\title{
Exploring MAORY performances through tolerance analysis
}

\author{
Mauro Patti*ab ${ }^{\text {ab }}$ Matteo Lombini ${ }^{\mathrm{b}}$, Emiliano Diolaiti $^{\mathrm{b}}$, Fausto Cortecchia $^{\mathrm{b}}$, Paolo Ciliegi $^{\mathrm{b}}$ \\ aAlma Mater Studiorum, Università di Bologna, Dipartimento di Fisica e Astronomia, Viale Berti Pichat 6/2, Bologna, \\ IT 40127; \\ bINAF - Osservatorio Astronomico di Bologna, Via Piero Gobetti 93/3, Bologna, IT 40129;
}

\begin{abstract}
MAORY is the E-ELT first-light AO module that aims to provide multi-conjugate and single-conjugate adaptive optics compensation for the MICADO high-angular resolution infrared camera and spectrograph. The system performances must achieve diffraction limited image quality and very low geometric distortion. The goal of this paper is to identify, from a purely opto-mechanical point of view, the manufacturing and integration errors that could affect the astrometry and wavefront error requirements. We have performed a preliminary tolerance analysis on two optical designs that differ from having pure conical mirrors or non-zero aspherical terms for mirror surfaces. Following a Monte Carlo approach, we focused on the analysis of the expected performances, defining the advantages of the alternative design with aspherical terms for mirror surfaces.
\end{abstract}

Keywords: Extremely Large Telescope, Multi-conjugate Adaptive Optics, Tolerance analysis, Distortion, Astrometry

\section{INTRODUCTION}

MAORY [1][2] will be the multi-conjugate adaptive optics module for the European Extremely Large Telescope (EELT) [3] first light. MAORY is a post focal relay optics and has to enable high performance astronomical observations in particular by the MICADO [4] (MCAO Imaging Camera for Deep Observations) imager and spectrograph. To support the MICADO near-infrared camera, MAORY provides two adaptive optics modes. The first is a multi-conjugate adaptive optics (MCAO) mode; the second is a single conjugate adaptive optics (SCAO) mode.

At first light, MAORY will contain a single deformable mirror with provision for a second deformable mirror as an upgrade. In the MCAO mode, at least one deformable mirror within MAORY works together with the telescope M4. The two or more deformable mirrors (DMs) are conjugated to different altitudes to provide a wide field of view with high Strehl ratio and uniformity of the point spread function (PSF). The SCAO mode will deliver high Strehl ratio for a smaller field, rather than uniform Strehl ratio over a wide field. To deliver the performance required by the MICADO science case, MAORY uses six laser guide star (LGS) wavefront sensors (WFS) and three natural guide star (NGS) wavefront sensors in the MCAO mode, while the SCAO mode, as defined, works on a single bright star.

The current baseline optical design of MAORY is based on six mirrors (including one or two adaptive mirrors) and a dichroic beam-splitter. This dichroic beam-splitter is used to split the science light from the LGS light that is transmitted to the LGS WFSs by means of a focusable objective [5].

The MAORY performances and tolerance analysis, described in this paper, are referred to the science path excluding any optics of the LGS objective.

In section 2, we summarize the principal requirements that lead the instrument design and constrain the tolerance analysis. Section 3 describes two optical design under development and shows the differences in terms of nominal performances of the two solutions. Section 4 describes the logic behind the tolerance analysis and shows the expected performances of MAORY perturbed by opto-mechanical tolerances. 


\section{MAORY OPTICAL DESIGN REQUIREMENTS}

The MAORY field of view (FoV) is 180 " diameter and has to re-image the E-ELT focal plane with diffraction limited quality and low geometric distortion [6]. The optics are optimized for the spectral band between $0.8 \mu \mathrm{m}$ and $2.4 \mu \mathrm{m}$ and two exit ports will be fed. The first one is placed $1800 \mathrm{~m}$ below the optical axis and it is the entrance port for MICADO on a gravity invariant configuration. The second exit port will feed an instrument that, at the time of writing, is not defined yet. The post focal DMs optical conjugates are planned to be at $4 \mathrm{~km}$ and $12.7 \mathrm{~km}$ altitude. As rule of thumb, the image quality should be better than 1/10 of actuator pitch at DMs conjugate. The MAORY exit pupil ellipticity shall be < $1 / 400$ of the pupil diameter, at any position in the technical FoV of the NGS sources. In the MICADO FoV of 53"x 53", MAORY must deliver diffraction limited optical quality and very low field distortion. Considering a narrowband filter, MICADO can integrate for 120 seconds within a single exposure. Considering a zenith avoidance of $10^{\circ}$, the maximum field rotation is $\approx 79 \mathrm{arcsec} / \mathrm{s}$ at E-ELT site latitude. During this rotation, the FWHM of the long-exposure PSF due to the MAORY optics should not increase by more than $10 \%$ of its nominal value. This means, for wavelength $\lambda=1 \mu \mathrm{m}$, the amount of distortion shall be $<8 \mu \mathrm{m}$ at the MAORY exit focal plane (assuming F/17.7 focal ratio).

Relative field distortion residual, over all timescales in the range of 1 hour to 5 years, shall be $<50 \mu$ as (goal $<10 \mu$ as) in a 20 " FoV (goal 53" x 53" FoV) after $\mathrm{n}^{\text {th }}$ order transformation, comprehending all error sources.

\section{MAORY OPTICAL DESIGN OPTIONS}

At the time of writing, two optical design are object of study. They manly differ from having pure conical mirrors or non-zero aspherical terms for mirror surfaces. The light through the science path of MAORY is shown in Figure 1. All mirrors, except M11, have optical power. Mirror M11 is shown in two copies: M11a and M11b, corresponding to two different exit ports. Mirror M11b is deployable: it is normally out of the beam to let the light propagate to mirror M11a. In the alternative design, all mirrors except the two DMs (M8 and M9) and M11 are even aspheric surfaces with nonzero coefficient until the $8^{\text {th }}$ order. The LGS/NGS dichroic beam-splitter is positioned close to a pupil image while the two DMs, are optically conjugated to $12.7 \mathrm{~km}$ and $4 \mathrm{~km}$ respectively. These two mirrors M8 and M9 should be intended as rigid mirrors for the scope of this paper. In fact, in a partial implementation of the instrument, at least the deformable mirror M9 would be replaced by a rigid mirror. Moreover, it is of interest to evaluate the feasibility of mirror M8, for instrument test purposes. M10 reproduces the exit focal plane that is folded downward by M11 flat mirror to create a gravity invariant port for MICADO. In the current design, the DMs are concave and they have the same curvature radius and conic constant. The optical quality of the layer images on the DMs, in terms of Root-Mean-Square (RMS) spot radius, is lower than $1 / 10$ of actuator pitch. The focal aperture at the exit port is very similar to the E-ELT one $(\approx F 17.7)$.

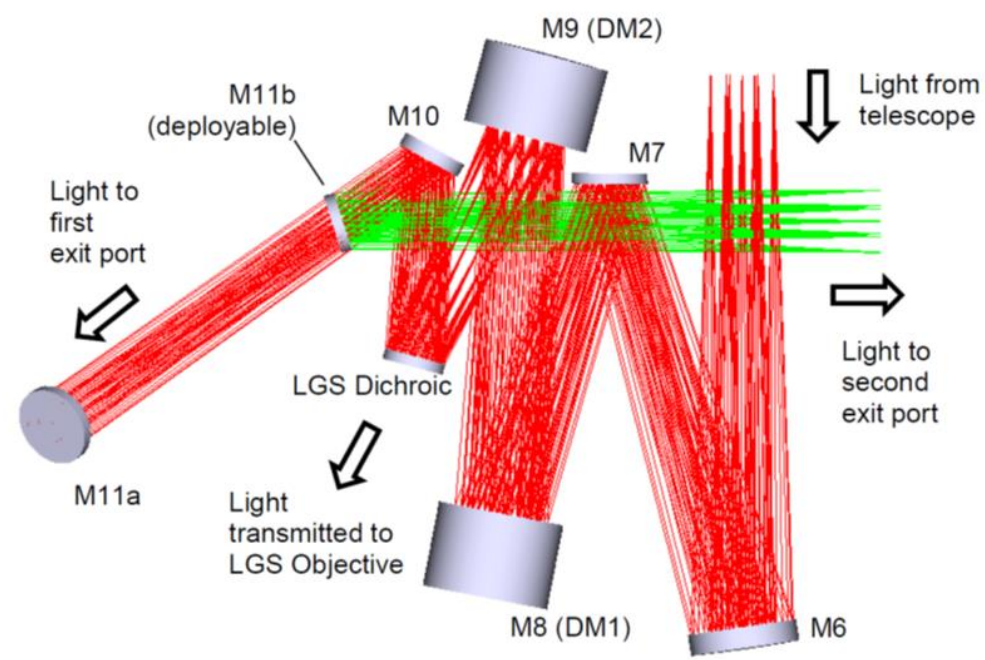

Figure 1: MAORY main path optical design 
Before a detailed description of tolerance analysis, the two optical design options were compared, in terms of nominal performances, by following the criteria listed here:

1. Wavefront Error (WFE) in the circle containing MICADO FoV (75" diameter)

2. WFE in the NGS FoV (annular, 180" < FoV < 75")

3. Astrometric residual error after the 3rd and 4th (5th if needed) order coordinates transformation in the circle containing the MICADO FoV (for a range of field rotations)

4. Star centroids movement after the maximum rotation for a single astrometric image in the circle containing the MICADO FoV

5. Star centroid movements due to optical distortion in the NGS FoV (for a range of field rotations)

The two designs have a very similar RMS WFE in terms of absolute values. The off-axis mirrors are decentered and tilted in one axis only, hence the WFE map is symmetric along the other axis. RMS WFE maps of MICADO FoV and NGS FoV are shown in Figure 2. They refer to MAORY design with even aspheric terms for mirror surface.
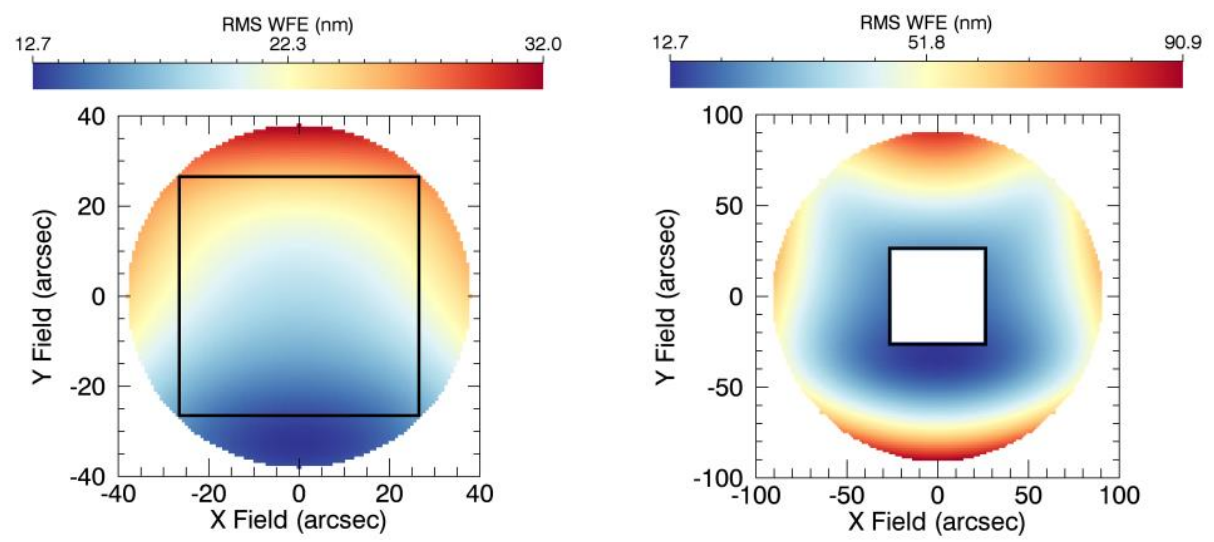

Figure 2: RMS WFE maps of MICADO FoV (left) and technical FoV (right). The black square is the 53"x53" MICADO FoV

Optical distortion is the main difference between the two design. Figure 3 shows (for both designs) the star centroids movements in the MICADO FoV for 120 seconds of single exposure. Figure 4 shows (for both designs) star centroids movement due to optical distortion in the NGS FoV. These distortions introduce an error in the positioning of NGS WFS probe known as plate scale error that adds differential tip-tilts to the MCAO loop and has to be calibrated.
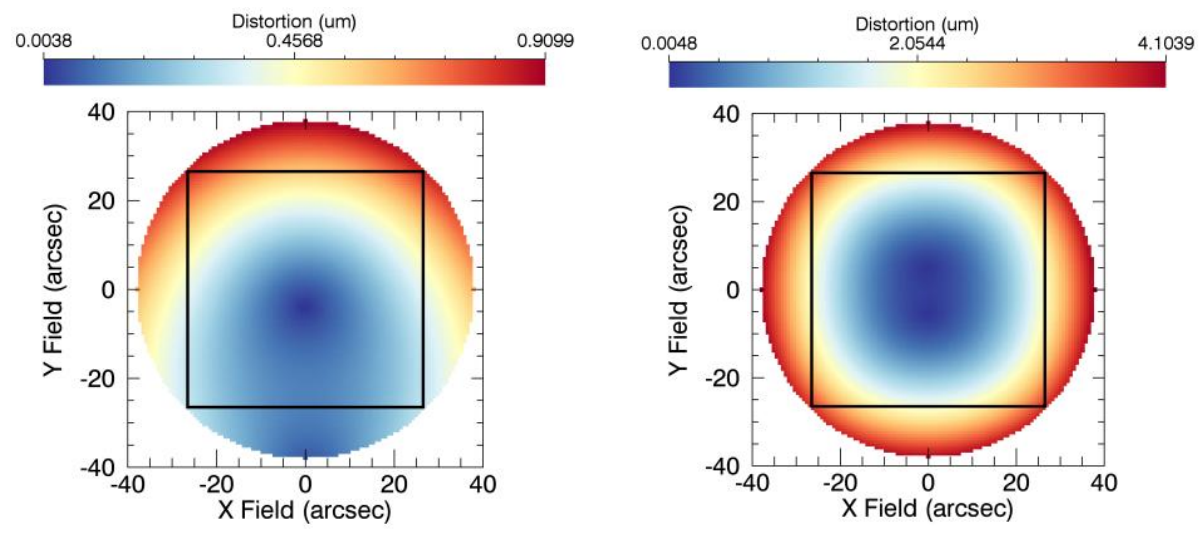

Figure 3: Star centroids movement for a single astrometric image in the circle containing the MICADO FoV (black square). Left plot refers to the design with even aspheric terms for mirror surfaces. Right plot refers to pure conical mirrors 

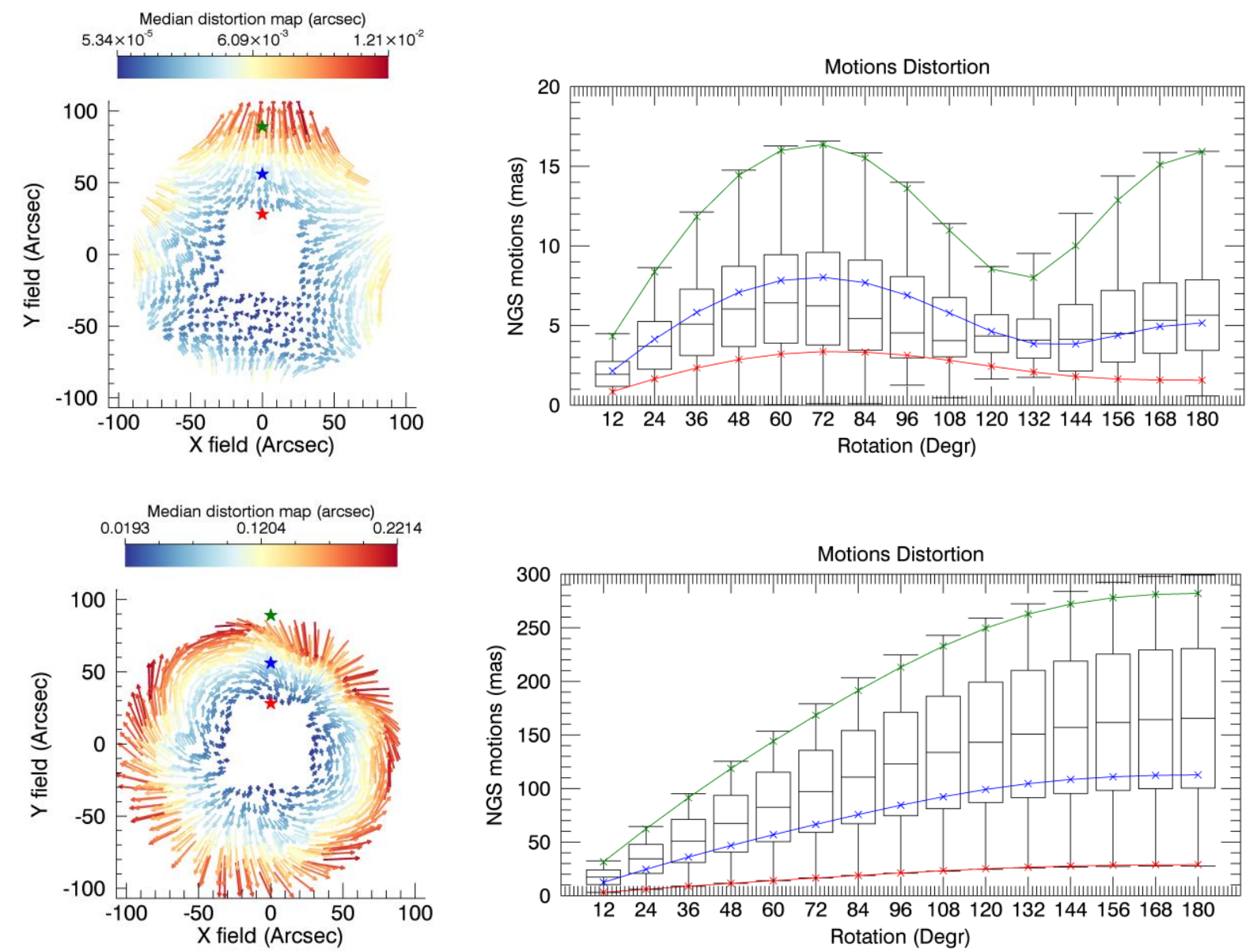

Figure 4: Star centroid movements (for a range of field rotations) due to optical distortion in the NGS FoV. First row refers to the design with even aspheric terms for mirror surfaces. Second row refers to pure conical mirrors. Distortion maps on the left are median of the considered field rotation angles. Box plots on the right show minimum and maximum values and quartiles of star centroid movements in the FoV

The positions of a set of $\mathrm{N}$ test stars placed over a regular square grid has been used to evaluate the astrometric residual error. Let's define $X_{\text {inp }}, Y_{\text {inp }}$ as initial coordinates of star centroids. $X_{0}, Y_{0}$ are coordinates of the star centroids after a given FoV rotation angle. Considering a range of field rotations in the circle containing the MICADO FoV, the distortion has been estimated and corrected using a $\mathrm{n}^{\text {rd }}$ order polynomial of the form:

$$
\begin{aligned}
& X=\Sigma_{\mathrm{i}, \mathrm{j}} K x_{i, j} \cdot X_{\mathrm{o}}{ }^{\mathrm{i}} \cdot Y_{\mathrm{o}}{ }^{\mathrm{j}} \\
& Y=\Sigma_{\mathrm{i}, \mathrm{j}} K y_{i, j} \cdot X_{\mathrm{o}}{ }^{\mathrm{i}} \cdot Y_{\mathrm{o}}{ }^{\mathrm{j}}
\end{aligned}
$$

Where $3^{\text {rd }}$ and $4^{\text {th }}\left(5^{\text {th }}\right.$ if needed) order indicates the max of the sum of the exponents $i$ and $j$ in equations. The $K x_{i, j}$ and $K y_{i, j}$ are $(\mathrm{n}+1) \mathrm{x}(\mathrm{n}+1)$ matrixes used to model the distortions introduced by the optics after a given FoV rotation angle. The $X, Y$ coordinates have been compared to $X_{i n p}, Y_{\text {inp }}$ in order to compute error and relative standard deviation. The final error in the position is the standard deviation computed as the quadratic sum of the $X$ and $Y$ error.

This is the astrometric residual error showed in Figure 5 for a range of field rotations angle. A $4^{\text {th }}$ order polynomial is enough to model distortions despite the MAORY design. 

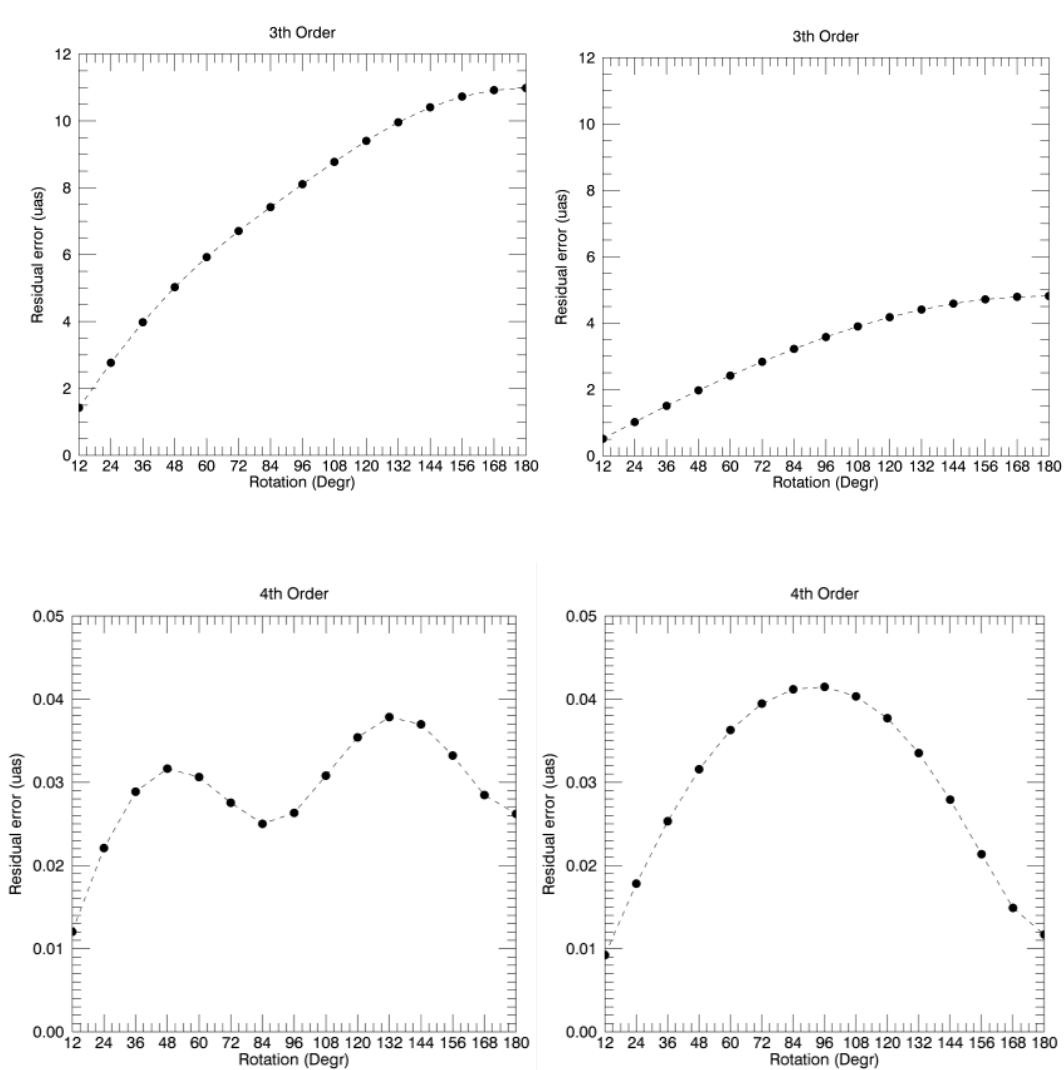

Figure 5: Astrometric residual errors for a range of field rotations in the circle containing the MICADO FoV. The error is the standard deviation computed as the quadratic sum of the $X$ and $Y$ error. First row: $3^{\text {rd }}$ order polynomial fit. Second row: $4^{\text {th }}$ order polynomial fit. First column: design with even aspheric terms for mirror surfaces. Second row: design with pure conical mirrors

The main properties and interfaces of the MAORY optical relay described in this paper are summarized in Table 1.

Table 1: MAORY main properties

\begin{tabular}{|c|c|c|}
\hline Surface & Diameter range depending on design (mm) & Proprieties \\
\hline M6 & $1100-1200$ & Off-axis hyperboloid (Could have even aspheric terms) \\
\hline M7 & $700-750$ & Off-axis hyperboloid (Could have even aspheric terms) \\
\hline M8 (DM1) & $850-1150$ & On-axis hyperboloid \\
\hline M9 (DM2) & $750-1150$ & On-axis hyperboloid \\
\hline Dichroic & $600-620$ & Flat \\
\hline M10 & $600-680$ & Off-axis hyperboloid (Could have even aspheric terms) \\
\hline M11 & $800-900$ & Flat (elliptical) \\
\hline \multicolumn{2}{|r|}{ Parameter } & Value range depending on design \\
\hline \multicolumn{2}{|l|}{ F\# } & $\approx 17.7$ \\
\hline \multicolumn{2}{|l|}{ Max FoV } & 3 arcmin diameter \\
\hline \multicolumn{2}{|c|}{ Exit pupil position } & $8000-8900 \mathrm{~mm}$ (before focus) \\
\hline \multicolumn{2}{|c|}{ Exit focal plane position } & $1800 \mathrm{~mm}$ below entrance optical axis \\
\hline \multicolumn{2}{|c|}{ RMS wavefront error over MICADO FoV } & $20-23 \mathrm{~nm}$ (Average) \\
\hline \multicolumn{2}{|c|}{ *Max geometric distortion in the NGS FoV } & [ Plate Scale $\approx 0.3 \mathrm{arcsec} / \mathrm{mm}]$ \\
\hline \multicolumn{2}{|c|}{ *Geometric distortion in MICADO FoV } & $<1-4.5 \mu \mathrm{m}$ \\
\hline \multicolumn{3}{|c|}{$\begin{array}{l}\text { * Definition of geometric distortion: } \\
\mathrm{T}=\text { Maximum integration time for narrow band astrometric observations } \approx 120 \mathrm{~s} \\
\mathrm{~A}=\text { Maximum derotator angular velocity } \approx 13.7 \times\left(1 / \cos \left(80^{\circ}\right)\right) \approx 79 \mathrm{arcsec} / \mathrm{s} \\
\mathrm{T} \times \mathrm{A} \approx 2.6^{\circ}\end{array}$} \\
\hline
\end{tabular}




\section{TOLERANCE ANALYSIS}

Tolerance process of optical systems is one of the most important step in the instrument design since it involves complex relationships across fabrication, assembly and alignment of the system. The tolerance analysis is intended to ensure that MAORY requested performances are satisfied when the final assembled instrument is operative. At the end, the assignment of tolerances to the various opto-mechanical parameters should be a trade-off between final cost of the system and its resulting performances. This section describes the logic behind the tolerance analysis.

The method used to estimate tolerances takes care of compensation of errors during assembly/alignment procedure and uses a Root-Sum-Square (RSS) merit function to combine independent error contributions. The sensitivity analysis on system performances considers each tolerance individually and, defined a merit function, the introduced errors are combined by RSS to find the net effect of all the tolerances on the system. This method assumes that errors introduced by a given tolerance are statistically uncorrelated and allows to identify parameters which are highly sensitive to certain errors, such as surface curvature radii or decenters.

The sensitivities related only to available Degree-Of-Freedom (DOF) can be used during the alignment phase of the instrument since the worst offenders to system performances are also the best set of compensators for required adjustments.

There are two requirements that limit the allowable changes of opto-mechanical parameters. The mean RMS WFE and the optical distortion. The first must satisfy diffraction limit performance over the MICADO FoV while the second must satisfy high astrometric accuracy and precision (described in Section 3).

Considering the RMS WFE, the intent was to maintain a maximum difference respect to the nominal design below $30 \%$. The allowed change is a 'rule of thumbs' for the AO systems tolerance analysis and keeps the system below the diffraction limit of MICADO FoV. As criterion for tolerancing, the defined merit function considers the RMS WFE referred to star centroids and adds boundary constraints on geometric distortion in the MICADO FoV, as described in Table 1 (last row).

The tolerance analysis has been broken down into 4 blocks that consider different error sources of the optical elements:

Block 1: $\quad$ Curvature radii, conic constants and, if present, aspheric terms

Block 2: $\quad$ Low order RMS surface irregularities (from Zernike 4 to Zernike 11)

Block 3: $\quad$ High order RMS surface irregularities (form Zernike 12 to Zernike 120)

Block 4: $\quad$ Alignment errors

Each block introduces a certain amount of performance degradations despite the considered MAORY design with or without even aspheric terms for mirror surfaces, hence the computed tolerance values are equal for both designs.

The first two blocks listed above, are supposed to be compensated by a design re-optimization or during the instrument alignment.

Block 3 is a worst offender for astrometric requirements and needs a dedicated calibration process to fulfil desired performances.

Block 4 is strictly related to mechanical accuracy and precision of optical mounts.

To evaluate the impact of tolerances on astrometry, a Monte Carlo simulation is mandatory. This simulation generates a series of random systems which meets the specified tolerances. It is a realistic simulation of expected performance since all applicable tolerances are simultaneously and exactly considered.

For each block, a Monte Carlo simulation of 100 realization was run and the criteria listed in Section 3 were used to investigate the system performance degradations or variations.

The exit pupil image diameter, the focal aperture and the exit pupil distance variations have also been calculated. Their changes, for each tolerance block, are always less than $0.1 \%$. 


\subsection{Block 1 results}

These tolerances are supposed to be compensated by design re-optimization once precise measures on the considered parameters has been delivered. The companies in charge of components manufacturing should deliver the final product within the limit imposed by these tolerances. Once the optics are ready, precise measures of their final parameters can be used as nominal values and the system can be re-optimized by means of free DOF used as variables during the instrument design phase.

Computed tolerances are summarized in Table 2 together with their effect on RMS WFE and optical distortions.

Table 2: Tolerance on curvature radii, conic constants and, if present, aspheric terms. For flat mirrors, the tolerance is expressed in terms of distance on the normal from the surface to the center of the curvature ("Sagitta"). The estimated changes of WFE and distortion should be combined by RSS to their nominal values.

\begin{tabular}{|c|c|c|c|}
\hline & Curvature radius & Conic Constant & Aspheric terms (if present) \\
\hline Conical mirrors & $0.08 \%$ & $\pm 0.05 \%$ & $\pm 0.1 \%$ \\
\hline Flat mirrors & $\pm 633 \mathrm{~nm}(\Delta \mathrm{Sag})$ & - & - \\
\hline \multicolumn{3}{|c|}{ RMS WFE - Estimated change } & $\approx 10 \mathrm{~nm}$ (Average) \\
\hline \multicolumn{3}{|c|}{ Geometric distortion in MICADO FoV - Estimated change } & $\approx 0.15 \mu \mathrm{m}$ (Median) \\
\hline \multicolumn{3}{|c|}{ Max geometric distortion in the NGS FoV - Estimated change } & $\begin{array}{l}\approx 0.36 \text { mas (Median) } \\
\text { te Scale } \approx 0.3 \operatorname{arcsec} / \mathrm{mm}]\end{array}$ \\
\hline
\end{tabular}

Astrometry performances: Since performance degradations are independent from design, we show Monte Carlo results of MAORY with even aspheric terms for mirror surfaces. Monte Carlo trials follow a normal distribution for considered tolerance values and results are shown in Figure 6 and Figure 7. They should be compared to nominal values shown in Figure 3 and Figure 5. In Figure 6, box plots show minimum and maximum values and quartiles of Monte Carlo trials distribution for the astrometric residual error on a range of field rotation angle. In Figure 7 is shown the median 2D map of Monte Carlo trials and its distribution of star centroids movement after the maximum rotation for a single astrometric image in the circle containing the MICADO FoV. Median values are almost equal to those of nominal design, hence the effect of this tolerance block is negligible in terms of astrometric performances.
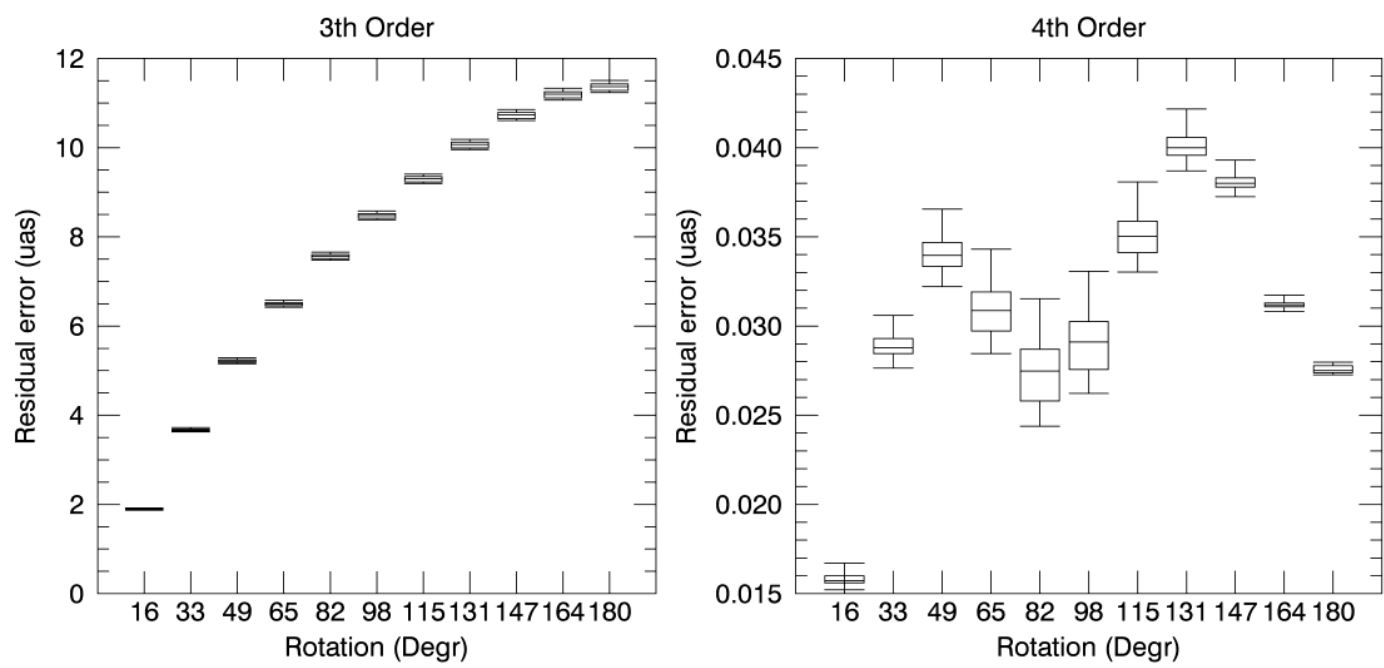

Figure 6: Astrometric residual errors for a range of field rotations in the circle containing the MICADO FoV. The error is the standard deviation computed as the quadratic sum of the $X$ and $Y$ error. Box plots show minimum and maximum values and quartiles of Monte Carlo trials distribution Since the considered tolerances introduce low order distortions, the distributions width is very narrow. 

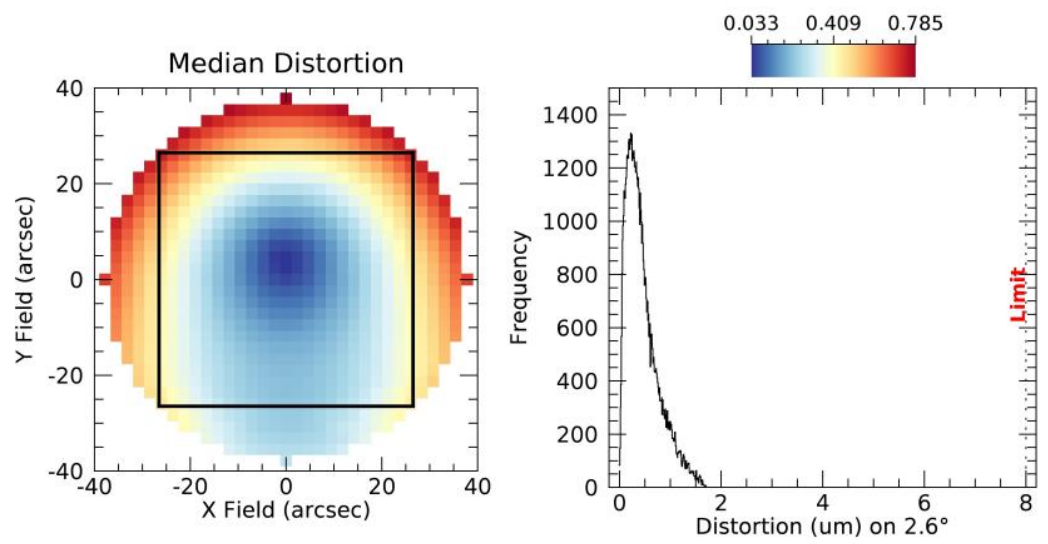

Figure 7: Star centroids movement for a single astrometric image in the circle containing the MICADO FoV (black square). Right: 2D distortion map of median values given by the Monte Carlo trials. Left: distribution of values given by the Monte Carlo trials.

\subsection{Block 2 results}

These tolerances are supposed to be compensated during the instrument alignment by means of the most sensitive DOF that have been chosen as the best set of compensators for required adjustments. Surface irregularities are modelled by means of standard Zernike coefficients whose max tolerance value is the exact RMS error of the surface. We verified that available DOF are able to compensate aberrations until Zernike coefficient 11 (the "spherical" term), hence the computed tolerances start from Zernike 4 (Z4 (the "defocus" term)) and end to Zernike 11 (Z11). Tip and Tilt terms (Z2 - Z3) are not considered because they are part of tolerance Block 4. Z4, although is part of curvature radii tolerance (Block 1), has to be considered for off-axis mirrors whose defocus term must be centered on the real footprint instead of the mirror vertex. On-axis surfaces tolerate Z4 as extra defocus (e.g. coating stress) in addition to that introduced by tolerance on curvature radii.

Computed tolerances are summarized in Table 3 together with their effect on RMS WFE and optical distortions.

Table 3: Low order RMS surface irregularities. The estimated changes of WFE and distortion should be combined by RSS to their nominal values.

\begin{tabular}{|c|c|c|c|c|c|c|c|c|}
\hline & $\mathbf{Z 4}(\mathbf{n m})$ & $\mathbf{Z 5}(\mathbf{n m})$ & $\mathrm{Z6}(\mathrm{nm})$ & $\mathbf{Z 7}(\mathbf{n m})$ & $\mathbf{Z 8}(\mathbf{n m})$ & $\mathbf{Z 9}(\mathbf{n m})$ & Z10 (nm) & Z11 (nm) \\
\hline M6 & 15 & 7 & 9 & 9 & 7 & 6 & 9 & 5 \\
\hline M7 & 15 & 7 & 7 & 6 & 7 & 4 & 7 & 7 \\
\hline M8 & 25 & 6 & 6 & 6 & 7 & 4 & 7 & 7 \\
\hline M9 & 20 & 5 & 5 & 5 & 7 & 4 & 7 & 7 \\
\hline Dichroic & 25 & 4 & 5 & 4 & 4 & 4 & 5 & 5 \\
\hline M10 & 15 & 6 & 6 & 6 & 7 & 8 & 9 & 5 \\
\hline M11 & 20 & 7 & 7 & 7 & 7 & 7 & 7 & 7 \\
\hline \multicolumn{6}{|c|}{ RMS WFE - Estimated change } & \multicolumn{3}{|c|}{$\approx 20 \mathrm{~nm}$ (Average) } \\
\hline \multicolumn{6}{|c|}{ Geometric distortion in MICADO FoV - Estimated change } & \multicolumn{3}{|c|}{$\approx 0.09 \mu \mathrm{m}$ (Median) } \\
\hline \multicolumn{6}{|c|}{ Max geometric distortion in the NGS FoV - Estimated change } & \multicolumn{3}{|c|}{$\begin{array}{c}\approx 0.32 \text { mas (Median) } \\
{[\text { Plate Scale } \approx 0.3 \operatorname{arcsec} / \mathrm{mm} \text { ] }}\end{array}$} \\
\hline
\end{tabular}

Results of the Monte Carlo trials in terms of astrometric performances are shown in Figure 8 and Figure 9. Refer to Section 4.1 for the meaning of the plots. 

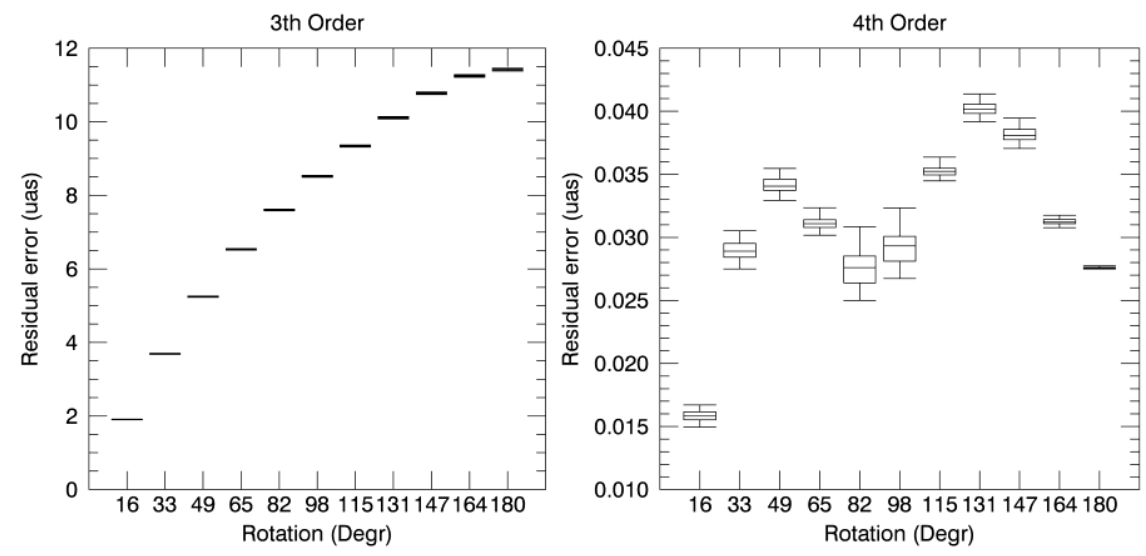

Figure 8: Astrometric residual errors for a range of field rotations in the circle containing the MICADO FoV. The error is the standard deviation computed as the quadratic sum of the $X$ and Y error. Box plots show minimum and maximum values and quartiles of Monte Carlo trials distribution. Since the considered tolerances introduce low order distortions, the distributions width is very narrow.
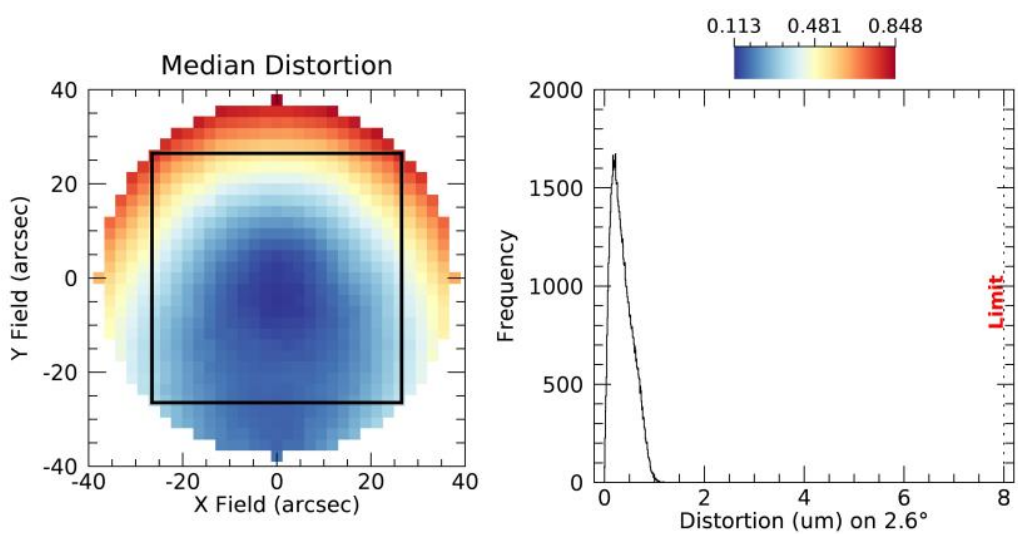

Figure 9: Star centroids movement for a single astrometric image in the circle containing the MICADO FoV (black square). Right: $2 D$ distortion map of median values given by the Monte Carlo trials. Left: distribution of values given by the Monte Carlo trials.

\subsection{Block 3 results}

These tolerances cannot be compensated by means of components DOF. The high order surface irregularities, in addition to WFE, cause astrometric residual errors that are large compared to those introduced by other tolerances. Surface irregularities are modelled by means of standard Zernike coefficients whose max tolerance value is the exact RMS error of the surface. The Zernike terms greater than 11 (the "spherical" term) are set to a value so that the RSS of the coefficients yields the specified RMS value.

Considering different field points, their beam footprints overlaps differently on each MAORY mirror surface. As the field rotates during the observations, the beam footprints cross the static irregularities on each mirrors surface. The lower is the overlap of different footprints, the bigger is the difference in terms of irregularities introduced in each optical trail relative to a given field point. As the optical footprint on a surface is the smaller the farther the surface is from the pupil plane, this error is largest for the surfaces with the largest conjugate ranges. For MAORY, this means that it is largest for the instrument selection mirror (M11) followed by the first mirror after the entrance focal plane (M6). Our results show that $12 \mu$ as residuals should be achievable with realistic requirements for the surface qualities and calibration time. This is not a hard limit as it can be reduced with on-sky observations of more objects and/or for longer times.

Computed tolerances are summarized in Table 4 together with their effect on RMS WFE and geometric distortions. 
Table 4: High order RMS surface irregularities. The estimated changes of WFE and distortion should be combined by RSS to their nominal values.

\begin{tabular}{|c|c|}
\cline { 2 - 3 } \multicolumn{1}{c|}{} & \multicolumn{2}{c|}{ Square root of the sum from Z12 to Z120 } \\
\hline All surfaces & $10 \mathrm{~nm}$ \\
\hline RMS WFE - Estimated change & $\approx 12 \mathrm{~nm}$ (Average) \\
\hline Geometric distortion in MICADO FoV - Estimated change & $\approx 0.26 \mu \mathrm{m}$ (Median) \\
\hline Max geometric distortion in the NGS FoV - Estimated change & $\begin{array}{c}\approx 0.1 \text { mas (Median) } \\
{[\text { Plate Scale } \approx 0.3 \text { arcsec/mm ] }}\end{array}$ \\
\hline
\end{tabular}

Results of the Monte Carlo trials in terms of astrometric performances are shown in Figure 10 and Figure 11 . Refer to Section 4.1 for the meaning of the plots. Because of high order effects previously described, in this case with need a $5^{\text {th }}$ order polynomial fit to minimize the astrometric residual error. Figure 10 also reports the only contribution of M11 high order surface irregularities. M11, which is close to the MAORY focal plane, is the worst offender in terms of astrometric residuals and its contribution to the total error is about $75 \%$.
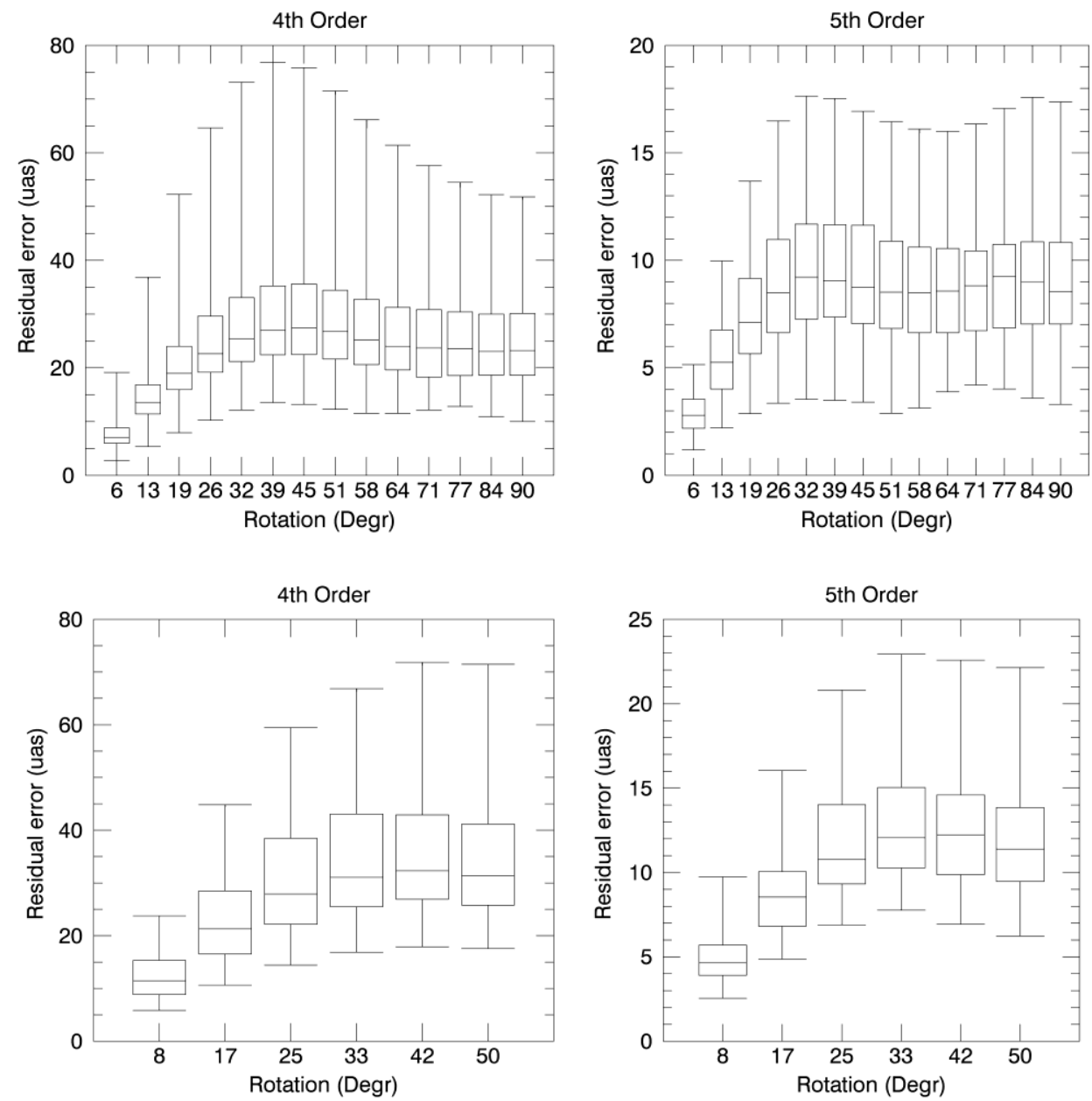

Figure 10: Astrometric residual errors for a range of field rotations in the circle containing the MICADO FoV. The error is the standard deviation computed as the quadratic sum of the $X$ and $Y$ error. Box plots show minimum and maximum values and quartiles of Monte Carlo trials distribution. Since the considered tolerances introduce high order distortions, the distributions width is larger than that seen in other tolerance block results. Upper plots refer to the only contribution of M11 high order surface irregularities. Lower plots refer to high order surface irregularities of all mirrors. In this case, we show values for a shorter range of field rotations since around 30 degree the residual error reach a plateau. 

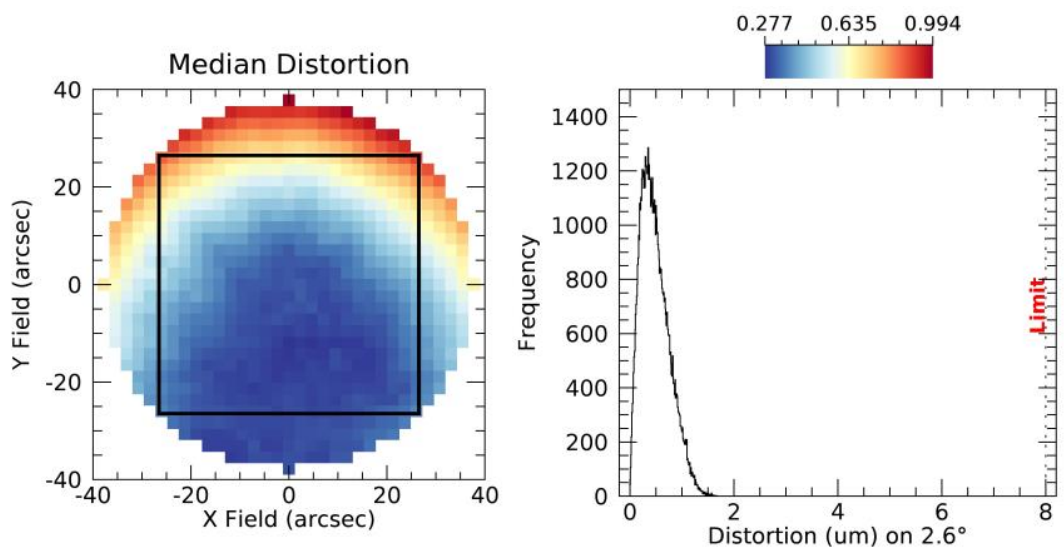

Figure 11: Star centroids movement for a single astrometric image in the circle containing the MICADO FoV (black square). Right: 2D distortion map of median values given by the Monte Carlo trials. Left: distribution of values given by the Monte Carlo trials.

\subsection{Block 4 results}

These tolerances refer to mechanical accuracy and precision of optical mounts. Sensitivity analysis on DOF tolerance of MAORY is a crucial step to understand the coupling between component misalignments and their effect on system performance. The DOF, used as compensators of misalignments, are rigid body motions of each optical surface that can be controlled by active components (i.e. a hexapod structure) during operations. These are:

- $\quad$ TT in $x$ and $y$ axes

- Decenter in $\mathrm{x}$ and $\mathrm{y}$ axes

- Axial position

The active compensators should work in combination with a metrology system that provide the necessary information to change the optical path according to estimate misalignments.

Computed tolerances are summarized in Table 5 together with their effect on RMS WFE and optical distortions. During the instrument alignment, available DOF have to move within these values in terms of repeatability. This is also the stability that each mirror has to reach in night-time.

Table 5: Tolerance on surface DOF. The estimated changes of WFE and distortion should be combined by RSS to their nominal values.

\begin{tabular}{|c|c|c|c|}
\hline & Tilts & Decenters & Axial position \\
\hline All mirrors & $\pm 17.5 \mu \mathrm{rad}$ & $\pm 50 \mu \mathrm{m}$ & $\pm 50 \mu \mathrm{m}$ \\
\hline \multicolumn{3}{|c|}{ RMS WFE - Estimated change } & $\approx 25 \mathrm{~nm}$ (Average) \\
\hline \multicolumn{3}{|c|}{ Geometric distortion in MICADO FoV - Estimated change } & $\approx 1.2 \mu \mathrm{m}$ (Median) \\
\hline \multicolumn{3}{|c|}{ Max geometric distortion in the NGS FoV - Estimated change } & $\begin{array}{c}\approx 0.42 \text { mas (Median) } \\
\text { [ Plate Scale } \approx 0.3 \operatorname{arcsec} / \mathrm{mm}]\end{array}$ \\
\hline
\end{tabular}

In this case, the Monte Carlo simulation considers a parabolic distribution since it yields selected values that are more likely to be at the extreme ends of the tolerance range. Results of the simulation in terms of astrometric performances are shown in Figure 12 and Figure 13. Refer to Section 4.1 for the meaning of the plots. We can say that, by using a polynomial fit greater than $2^{\text {nd }}$ order, the astrometric residual error is independent from misalignments since the distributions width of Monte Carlo trials is close to zero (see Figure 12). 

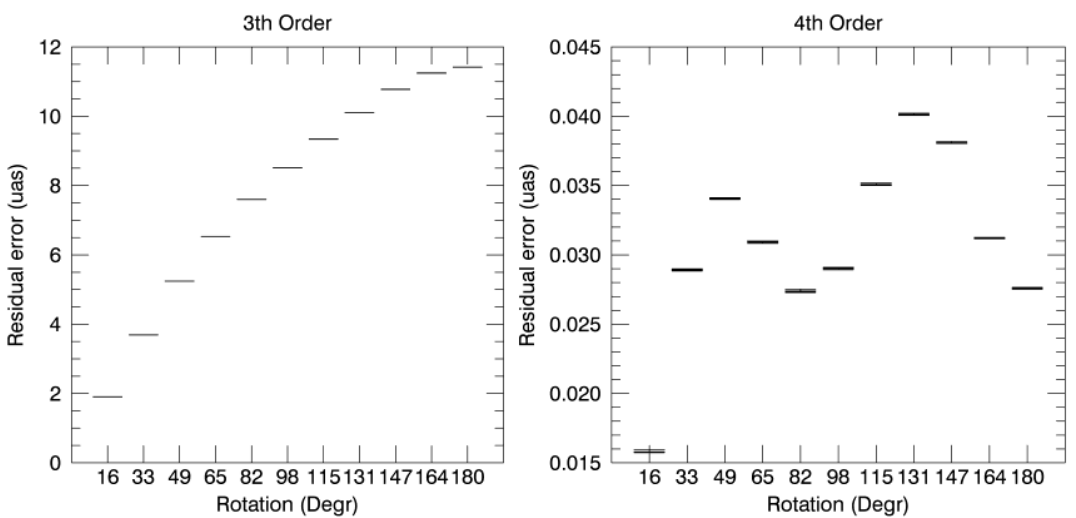

Figure 12: Astrometric residual errors for a range of field rotations in the circle containing the MICADO FoV. The error is the standard deviation computed as the quadratic sum of the $X$ and Y error. Since the considered tolerances introduce very low order distortions, the distributions width of Monte Carlo trials is close to zero. This means the astrometric residual error is independent from misalignments.
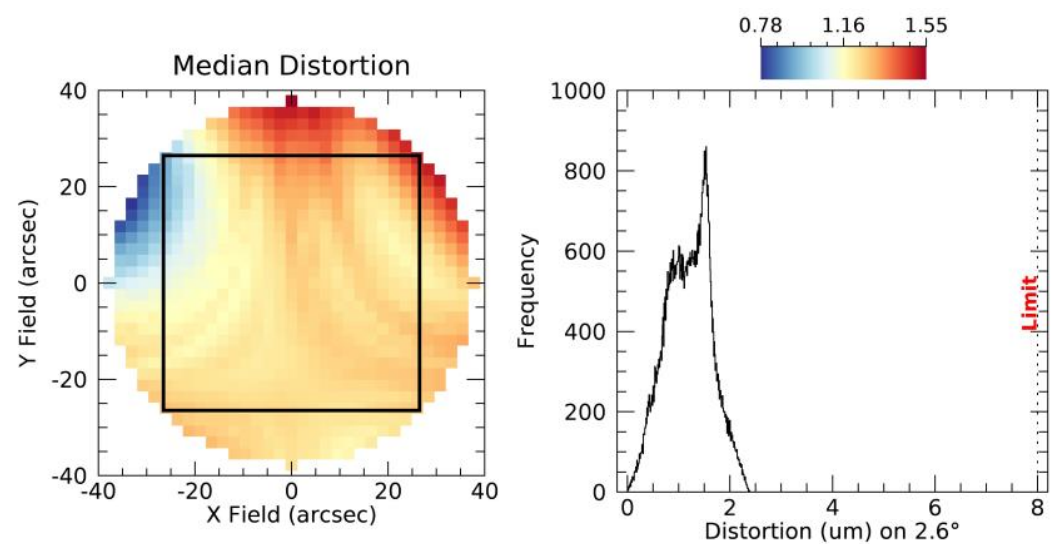

Figure 13: Star centroids movement for a single astrometric image in the circle containing the MICADO FoV (black square). Right: $2 D$ distrortion map of median values given by the Monte Carlo trials. Left: distribution of values given by the Monte Carlo trials.

\section{CONCLUSIONS}

The performances of two optical design of MAORY has been presented as well as the logic behind the tolerance analysis. With the computed tolerance, both optical designs fulfill the requirements. The main difference, in terms of performances, between the two design is the geometric distortion. That means star centroid moves due to optical distortion in the MAORY FoV for a range of field rotations. In terms of absolute values, at the edge of the field, distortions introduced by the optical design with even aspheric terms for mirror surface, are one order of magnitude lower than that relative to pure conical mirrors. It is possible to calibrate these distortions by polynomial fit and the residuals are independent from the considered design. This is a first step to develop detailed strategies to deal with distortion calibration in the NGS and MICADO FoV. The final choice about MAORY design will be a trade-off between cost of aspheric mirrors and performances.

\section{ACKNOWLEDGMENTS}

This work has been partly supported by the "Progetto Premiale E-ELT" funded by the Italian Ministero dell'Istruzione, dell'Università e della Ricerca, and by the European Community Framework Program 7 (OPTICON project, grant agreement no. 312430). 


\section{REFERENCES}

[1] Diolaiti, E., "MAORY: A Multi-conjugate Adaptive Optics RelaY for the E-ELT," The Messenger 140, 28-29 (2010).

[2] Diolaiti, E.; Arcidiacono, C.; Bregoli, G.; Butler, R. C.; Lombini, M.; Schreiber, L.; Baruffolo, A.; Basden, A.r; Bellazzini, M.; Cascone, E.; Ciliegi, P.; Cortecchia, F.; Cosentino, G.; De Caprio, V.; De Rosa, A.; Dipper, N.; Esposito, S.; Foppiani, I.; Giro, E.; Morgante, G.; Myers, R.; Patru, F.; Ragazzoni, R.; Riccardi, A.; Riva, M.; Zerbi, F. M.; Casali, M.; Delabre, B.; Hubin, N.; Kerber, F.; Le Louarn, M.; Marchetti, E.; Ramsay, S.; Stroebele, S.; Vernet, E.; "Preparing for the phase B of the E-ELT MCAO module project."; Proceedings of the SPIE, Volume 9148, id. 91480Y, (2014);

[3] Gilmozzi, R. and Spyromilio J., "The 42m European ELT: status," in Society of Photo-Optical Instrumentation Engineers (SPIE) Conference Series, Society of Photo-Optical Instrumentation Engineers (SPIE) Conference Series 7012, (2008).

[4] Davies, R.; Schubert, J.; Hartl, M.; Alves, J.; Clénet, Y.; Lang-Bardl, F.; Nicklas, H.; Pott, J.-U.; Ragazzoni, R.; Tolstoy, E.; Agocs, T.; Anwand-Heerwart, H.; Barboza, S.; Baudoz, P.; Bender, R.; Bizenberger, P.; Boccaletti, A.; Boland, W.; Bonifacio, P.; Briegel, F.; Buey, T.; Chapron, F.; Cohen, M.; Czoske, O.; Dreizler, S.; Falomo, R.; Feautrier, P.; Förster Schreiber, N.; Gendron, E.; Genzel, R.; Glück, M.; Gratadour, D.; Greimel, R.; Grupp, F.; Häuser, M.; Haug, M.; Hennawi, J.; Hess, H.-J.; Hörmann, V.; Hofferbert, R.; Hopp, U.; Hubert, Z.; Ives, D.; Kausch, W.; Kerber, F.; Kravcar, H.; Kuijken, K.; Lang-Bardl, F.; Leitzinger, M.; Leschinski, K.; Massari, D.; Mei, S.; Merlin, F.; Mohr, L.; Monna, A.; Müller, F.; Navarro, R.; Plattner, M.; Przybilla, N.; Ramlau, R.; Ramsay, S.; Ratzka, T.; Rhode, P.; Richter, J.; Rix, H.-W.; Rodeghiero, G.; Rohloff, R.-R.; Rousset, G.; Ruddenklau, R.; Schaffenroth, V.; Schlichter, J.; Sevin, A.; Stuik, R.; Sturm, E.; Thomas, J.; Tromp, N.; Turatto, M.; Verdoes-Kleijn, G.; Vidal, F.; Wagner, R.; Wegner, M.; Zeilinger, W.; Ziegler, B.; Zins, G. "MICADO: first light imager for the E-ELT", Proceedings of the SPIE, volume 9908 id 73, (2016);

[5] M. Lombini ; A. De Rosa ; P. Ciliegi ; F. Cortecchia ; E. Diolaiti ; Mauro Patti ; M. Bonaglia ; L. Busoni ; V. De Caprio ; S. Esposito ; P. Feautrier ; P. Rabou ; M. Riva ; E. Stadler; "Optical design of the post-focal relay of MAORY”. Proc. SPIE 9908, Ground-based and Airborne Instrumentation for Astronomy VI, 9908AB (August 9, 2016);

[6] Trippe, S.; Davies, R.; Eisenhauer, F.; Schreiber, N. M. Förster; Fritz, T. K.; Genzel, R.; "High-precision astrometry with MICADO at the European Extremely Large Telescope"; Monthly Notices of the Royal Astronomical Society, Volume 402, Issue 2, pp. 1126-1140, (2010); 